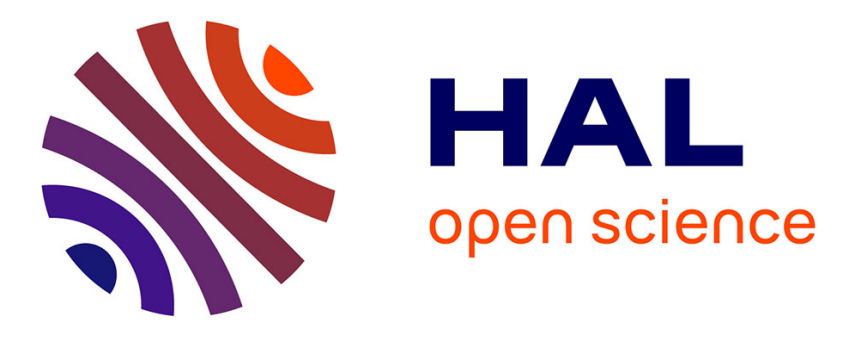

\title{
An intimate virtual counselor for a better user experience
}

\author{
Delphine Potdevin, Nicolas Sabouret, Céline Clavel
}

\section{To cite this version:}

Delphine Potdevin, Nicolas Sabouret, Céline Clavel. An intimate virtual counselor for a better user experience. 20th ACM International Conference on Intelligent Virtual Agents (IVA '20), Oct 2020, Glasgow (en ligne), United Kingdom. pp.16, 10.1145/3383652.3423859 . hal-03195531

\section{HAL Id: hal-03195531 \\ https://hal.science/hal-03195531}

Submitted on 11 Apr 2021

HAL is a multi-disciplinary open access archive for the deposit and dissemination of scientific research documents, whether they are published or not. The documents may come from teaching and research institutions in France or abroad, or from public or private research centers.
L'archive ouverte pluridisciplinaire HAL, est destinée au dépôt et à la diffusion de documents scientifiques de niveau recherche, publiés ou non, émanant des établissements d'enseignement et de recherche français ou étrangers, des laboratoires publics ou privés. 


\title{
An intimate virtual counselor for a better user experience
}

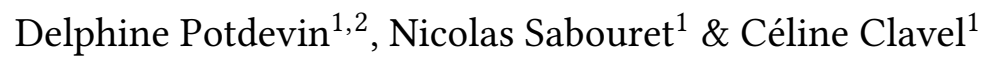 \\ name.lastname@limsi.fr \\ ${ }^{1}$ Université Paris-Saclay, LIMSI-CNRS \\ ${ }^{2}$ DAVI-Les Humaniseurs
}

\begin{abstract}
Embodied Conversational Agents (ECAs) are suffering from a lack of adoption from users. This paper emphasizes virtual intimacy as a lever to support social dimension of human-agent interaction and enhance user experience. Here, we present an autonomous virtual counselor, expert in tourism, which provides multimodal intimacyrelated behaviors. Real tourists interacting in the wild with our intimate agent only perceived its honesty and genuineness, which was insufficient to enhance social presence and user experience. However, our results suggest that the perception of virtual intimacy could be a strong predictor of social presence and user experience. When perceived, virtual intimacy may be, then, an important mean to foster user experience and ECAs adoption.
\end{abstract}

\section{KEYWORDS}

Virtual Intimacy, Human-agent interaction, Multimodal behavior, Social presence, User Experience, Field conditions interactions

\section{ACM Reference Format:}

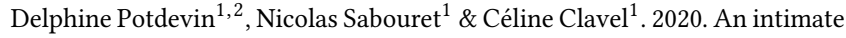
virtual counselor for a better user experience. In IVA '20: Proceedings of the 20th ACM International Conference on Intelligent Virtual Agents (IVA '20), October 19-23, 2020, Virtual Event, Scotland Uk. ACM, New York, NY, USA, 3 pages. https://doi.org/10.1145/3383652.3423859

\section{INTRODUCTION}

Embodied Conversational Agents (ECAs) have been used for online customer-relationship for almost two decades, in a wide range of domains such as retailing, healthcare, bank and insurance, education. However, studies show that they are still suffering from a lack of adoption from users [8]. Indeed, the task competencies of ECAs are not enough to satisfy customer-relationship. ECAs must also be provided with social competencies so as to be recognized as a social partner in human-agent interactions. This is referred to as social presence [17], i.e. the sense of being with someone else [16].

In this paper, we focus on one sub-dimension of social presence: intimacy [2], as a means for satisfying customer-relationship. This dimension has been mainly studied in interpersonal relationships. It is related to close and personal relationships existing between two partners [15]. Intimacy appeals to the overall of physical, behavioral, cognitive and emotional sharing experiences, and involves both verbal and nonverbal communication [14].

Permission to make digital or hard copies of part or all of this work for personal or classroom use is granted without fee provided that copies are not made or distributed for profit or commercial advantage and that copies bear this notice and the full citation on the first page. Copyrights for third-party components of this work must be honored For all other uses, contact the owner/author(s).

IVA '20, October 19-23, 2020, Virtual Event, Scotland Uk

(C) 2020 Copyright held by the owner/author(s).

ACM ISBN 978-1-4503-7586-3/20/09.

https://doi.org/10.1145/3383652.3423859
Intimacy has already been studied in relation with virtual agents. Researchers demonstrated that self-disclosing fosters the users' social perception of the agent [5]. Multimodal intimacy-related behaviors of a professional ECA were strongly perceived by external observers [13]. Moreover, Lee and Choi suggest that intimate ECAs increase users satisfaction [7] and give evidence that intimacy may represent a lever to ECAs adoption.

However, further investigations are required to explore the overall of user experience [11] when interacting with an ECAs. To this goal, we propose to rely on the MeCue model [10]. Among existing models in the literature, MeCue describes user experience as the overall of instrumental and non instrumental perceptions of the system, as well as the resulting emotions. It is one of the rare models to focus on emotions and appears as an appropriate framework to study user experience in human-ECA interactions.

We conducted an interactive experiment in field conditions, involving natural interactions between a virtual counselor and real tourists, to study the impact of an intimate ECA on the perception of virtual intimacy, social presence and user experience. To this end, we have built an autonomous virtual counselor expert in tourism, capable of informing and supporting visitors of a tourism office, but also to express multimodal intimate behaviors.

\section{INTERACTIVE STUDY}

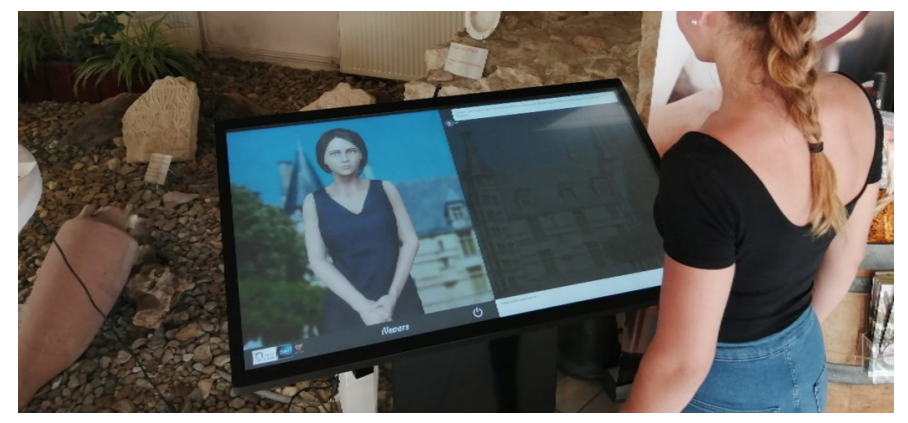

Figure 1: The interactive device supporting the interaction between our virtual counselor and real tourists.

\subsection{Experimental design}

Our experimental design included 2 between-subject conditions: the intimate condition involved an ECA with intimate behaviors, whereas the control condition involved an no emotional ECA. Intimate behaviors of the agent relied on a model of virtual intimacy for ECAs [13], which has been proven to be correctly identified by users. 
2.1.1 Verbal communication. Whatever the experimental condition, the virtual counselor provided tourists with task-related information. In intimate condition only, the agent provided additional intimate clues. These intimate behaviors were related to the expression of honesty and genuineness (e.g. self-disclosing, personal advice), involved positivity (e.g. positive feelings, commitment, personal recommendations), and testified of a mutual comprehension (e.g. reassuring, understanding, support) [13].

The virtual counselor was able to discuss about broad themes: she could indicate toilets location, give information on the artists who created the stained glass windows of the cathedral, or even provide recommendations on good restaurants. As a consequence, each answer of the intimate agent could be of a variable level of intimacy, both in terms of the quantity and quality of the social signs. The participants were free to discuss any topic with the agent and therefore, did not face the same intimate behaviors.

2.1.2 Nonverbal communication. Whatever the experimental condition, the virtual counselor was provided with "idle" behavior [3]: small variations of body posture, breathing moves, blinking and small variations of gaze orientation. In addition to idle motion, the agent's behavior varied according to 4 parameters (e.g. gestures, head moves, gaze orientation and facial expression of emotions), in the intimate and control condition. An animation engine have been specifically developed for the study and triggered the agent's animations in real time and in accordance to the agent's verbal content. Each response could involve concepts related to words or phrases and which triggered a specific nonverbal behavior. In absence of trigger words, non emotional behavior were displayed to maintain behavioral credibility.

In control condition, no concept were triggered and the agent's animation only included communicative gestures (e.g. descriptive or explaining), randomized gaze and did not involve head moves or emotional facial expressions. In intimate condition, concepts related to the expression of intimacy were triggered. Inspired from the literature $[1,12]$, these "intimate concepts" (e.g. "self") were associated with specific gestures (e.g. oriented toward the self), head moves (e.g. head tilts and nods), gaze and facial expressions of emotions (e.g. smiling).

\subsection{Procedure}

A total of 68 visitors (29 females) were recruited in a tourist office for the experiment, however only 60 participants were finally retained for technical reasons. All participants were of age $(M=41$, $S D=15.11, \operatorname{Min}=18, \operatorname{Max}=76)$ and were native french speakers. Visitors came spontaneously to the tourism office and were invited to participate in the experiment, by addressing their questions at first to the virtual counselor. Participants who accepted to participate were briefly introduced to the nature of the experiment and had to sign a consent form. In a second step, the experimenter introduced the device to participants, launched a new experimental session and gave instructions about the its use. Then, the experimenter left and subjects interacted with the agent by asking all the questions they wanted. When participants were done, the experimenter came back and stopped the experimental session. In a third step, participants had to complete an online survey. Participants were thanked and informed about the aim of the study. Participants were encouraged to ask additional tourist information to the office counselor.

\subsection{Material and measures}

The interactive device chosen for the experiment had a tactile screen, a microphone and load-speakers. The agent was developed by the company ${ }^{\circledR}$ DAVI and is provided with RETORIK technology: it benefits from a Natural Language Processing (NLP) system based on ontology and a sphere of knowledge related to tourism. The agent was provided with a synthetic voice and a text-to-speech system which enabled natural communication with users.

The online survey proposed to participants included 3 questionnaires assessing perception of virtual intimacy, social presence and user experience. We used, respectively, the Virtual Intimacy Scale (VIS) [13], as well as a french version of the Networking Minds [2] and the MeCue questionnaire [10] validated in french by [6]. The VIS included 15 items divided in 3 dimensions of honesty and genuineness, positivity, and "mutual comprehension". The Networking Minds presented 36 items and assessed social presence. The MeCue scale included 30 items distributed in 4 "units": Product perceptions, Emotions, Consequences and Global evaluation.

\section{RESULTS}

\subsection{Descriptive analysis}

In average, participants asked 11 requests $(S D=5.87)$ to the agent during the interaction. Globally, the agent could not understand $18 \%$ $(S D=15.15)$ of requests and did not provide a suitable response. We found no effect of the agent's misunderstanding on the perception of virtual intimacy, social presence and user experience. However, we found an impact of interaction length: although the effect on the "Emotions" unit is not significant $(F(1,58)=2.638, p=0.11$, $\left.R^{2}=0.44\right)$, we found a positive relationship between the number of questions and the perception of negative emotions $(F(1,58)=9.530$, $\left.p=0.003, R^{2}=0.14\right)$. Interestingly, this effect disappeared when we focused on the intimate condition, exclusively.

\subsection{Impact of intimacy related behaviors}

A first analysis studied the influence of the experimental condition on the perception of virtual intimacy. No difference was found between the mean score of virtual intimacy between intimate and control conditions $(t(58,2)=1.588, p=0.118, d=0.41, M=57.67$, $S D=12.72$ vs $M=52.35, S D=13.17)$. However, we focused on the factor Honesty and Genuineness and found a medium to large effect of the intimate condition $(t(58,2)=2.264, p=0.027, d=0.59$ $M=58.97, S D=12.33$ vs $M=50.56, S D=16.06$ ).

A second analysis studied the impact of the experimental condition on the perception of social presence. We found no effect of the agent's intimacy. However, found a medium of effect of virtual intimacy when we focused on the factor Copresence $(t(58,2)=2.178$, $p=0.033, d=0.56, M=5.32, S D=1.49$ vs $M=4.45, S D=1.62)$.

A third analysis studied the impact of the experimental condition on the perception of user experience. We found no effect of intimacy both on mean user experience and its constitutive units. We only found a strong effect of the intimate condition when we focused on the perception Status $(t(58,2)=2.520, p=0.015, d=0.65$, $M=3.08, S D=1.77$ vs $M=2.10, S D=1.22$ ). 


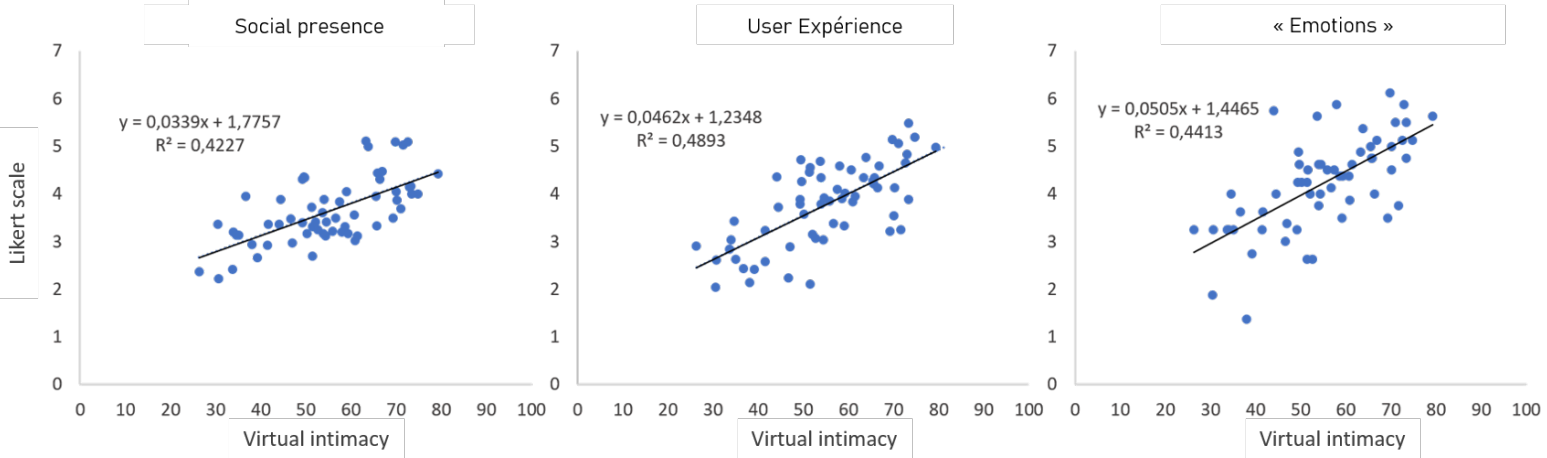

Figure 2: prediction of social presence (left), user experience (middle) and "Emotions" unit (right) according to virtual intimacy.

\subsection{Influence of the perceived virtual intimacy}

A first analysis of linear regression showed that perceived virtual intimacy significantly predicted the perception of social presence $(F(1,58)=42.472, p<0.001, \alpha=0.034, S E=0.005, \beta=0.650$ $\left.R^{2}=0.423\right)$, whatever the experimental condition. Two additional analyses, presented in Figure 2, showed that perceived virtual intimacy significantly predicted the perception of user experience $\left(F(1,58)=55.579, p<0.001, \beta=0.700, R^{2}=0.489\right)$ and its Emotions unit $\left(F(1,58)=45.817, p<0.001, \beta=0.664, R^{2}=0.441\right)$, whatever the experimental condition.

Following Mackinnon's method [9], we conducted a mediation analysis to study the impact of social presence on the effect of perceived virtual intimacy on user experience: a first multiple regression analysis $\left(F(2,57)=32.273, p<0.001, R^{2}=0.531\right)$ showed that only the perception of virtual intimacy $(t=4.748, \alpha=0.036$, $S E=0.008, p<0.001)$ remained a significant predictor of user experience, contrary to social presence that did not raised significance $(t=1.838, \alpha=0.271, S E=0.148, p=0.071)$. This result underlined the direct and independent effect of virtual intimacy on user experience and excluded the mediation effect of social presence.

\section{DISCUSSION}

This study explored the impact of intimacy related behavior displayed by a professional ECA on user's perceptions, in the wild Although previous work confirmed that virtual intimacy related behaviors could be perceived by external observers [13], we realized that social behaviors of the agent were not as well perceived by users in field conditions. First, self-disclosure - which is the most reported intimate behavior in the literacy- is associated with the honesty and genuineness dimension and may explain that this dimension has been particularly well perceived [15]. Moreover, positivity and mutual comprehension may have been expected as professional skills and may have been under-evaluated by users. Consequently, we failed to demonstrate an increased social presence and user experience with the intimate virtual counselor. However, this study identifies virtual intimacy as an interesting lever both for recognizing the agent as a social partner and fostering user experience. The perception of virtual intimacy appeared as a strong predictor of social presence and user experience. As in computer-mediated communication [2], virtual intimacy seems to be a consistent determinant of social presence in human-ECA interactions. Moreover, intimacy has a direct effect on user experience which suggests that specific socioemotional mechanisms, such as emotional contagion [4], may be involved and should be further explored.

\section{REFERENCES}

[1] Michael Argyle and Janet Dean. 1965. Eye-contact, distance and affiliation. Sociometry (1965), 289-304.

[2] Frank Biocca, Chad Harms, and Judee K Burgoon. 2003. Toward a more robust theory and measure of social presence: Review and suggested criteria. Presence: Teleoperators \& virtual environments 12,5 (2003), 456-480.

[3] Arjan Egges, Tom Molet, and Nadia Magnenat-Thalmann. 2004. Personalised real-time idle motion synthesis. In 12th Pacific Conference on Computer Graphics and Applications, 2004. PG 2004. Proceedings. IEEE, 121-130.

[4] Elaine Hatfield, John T Cacioppo, and Richard L Rapson. 1992. Primitive emotional contagion. Review of personality and social psychology 14 (1992), 151-177.

[5] Ivana Kruijff-Korbayová, Elettra Oleari, Anahita Bagherzadhalimi, Francesca Sacchitelli, Bernd Kiefer, Stefania Racioppa, Clara Pozzi, and Alberto Sanna. 2015. Young users' perception of a social robot displaying familiarity and eliciting disclosure. In International conference on social robotics. Springer, 380-389.

[6] Carine Lallemand and Vincent Koenig. 2017. How Could an Intranet be Like a Friend to Me?: Why Standardized UX Scales Don't Always Fit. In Proceedings of the European Conference on Cognitive Ergonomics 2017. ACM, 9-16.

[7] SeoYoung Lee and Junho Choi. 2017. Enhancing user experience with conversational agent for movie recommendation: Effects of self-disclosure and reciprocity. International fournal of Human-Computer Studies 103 (2017), 95-105.

[8] Ewa Luger and Abigail Sellen. 2016. Like having a really bad PA: the gulf between user expectation and experience of conversational agents. In Proceedings of the 2016 CHI Conference on Human Factors in Computing Systems. ACM, 5286-5297.

[9] David P MacKinnon, Ghulam Warsi, and James H Dwyer. 1995. A simulation study of mediated effect measures. , 41-62 pages.

[10] Sascha Mahlke. 2008. User experience of interaction with technical systems.

[11] Donald A Norman. 1991. Cognitive artifacts. Designing interaction: Psychology at the human-computer interface 1 (1991), 17-38.

[12] Magalie Ochs, Yu Ding, Nesrine Fourati, Mathieu Chollet, Brian Ravenet, Florian Pecune, Nadine Glas, Ken Prepin, Chloe Clavel, and Catherine Pelachaud. 2014. Vers des Agents Conversationnels Animés dotés d'émotions et d'attitudes sociales. Journal d'Interaction Personne-Système (FIPS) 3, 2, pp-1.

[13] Delphine Potdevin, Céline Clavel, and Nicolas Sabouret. 2018. Virtual Intimacy, this little something between us : a study about Human perception of intimate behaviors in Embodied Conversational Agents, In 18th International Conference on Intelligent Virtual Agents. Communication Research.

[14] Karen J Prager. 1995. Guilford series on personal relationships. The psychology of intimacy. New York: Guilford Press.

[15] Harry T Reis, Phillip Shaver, et al. 1988. Intimacy as an interpersonal process. Handbook of personal relationships 24, 3 (1988), 367-389.

[16] John Short, Ederyn Williams, and Bruce Christie. 1976. The social psychology of telecommunications. London: John Willey Sons, Ltd.

[17] Tibert Verhagen, Jaap Van Nes, Frans Feldberg, and Willemijn Van Dolen. 2014. Virtual customer service agents: Using social presence and personalization to shape online service encounters. Fournal of Computer-Mediated Communication 19, 3 (2014), 529-545. 